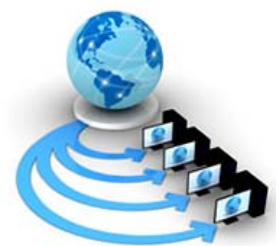

Volume 9, No. 1, January-February 2018

International Journal of Advanced Research in Computer Science

RESEARCH PAPER

Available Online at www.ijarcs.info

\title{
A CONCEPTUAL MODEL FOR UNDERSTANDING THE IMPACT OF TEXTUAL EMOTION MINING
}

\author{
Shivangi Chawla \\ Department of Computer Science, Jamia Millia Islamia \\ New Delhi, India
}

\author{
Monica Mehrotra \\ Department of Computer Science, Jamia Millia Islamia \\ New Delhi, India
}

\begin{abstract}
The widespread adoption of social media applications in today's internet world has created a revolution in the field of Textual emotion mining (TEM). TEM has gained a significant amount of interest in the past few years where a large community of researchers is focusing on the efficient extraction of emotions, ignoring the influential behavior of emotions. This paper presents a unique study to focus on the outcome of research on Textual Emotion Mining instead on the process of emotion mining. It classifies the output of TEM by presenting an emotion-output model. It also discusses the influential capability of emotions in various domains, shedding light on various new avenues by analyzing the different work done in this area.
\end{abstract}

Keywords: Emotion Mining; Social Emotions; Collective Emotions; Information Diffusion; Emotional Contagion

\section{INTRODUCTION}

Emotions form an inseparable part of human expression and with the advent of digital technologies and web 2.0, a large amount of user data is available online. User data in the form of text can potentially be extracted for emotions to benefit mankind in several commercial and social spheres like market analysis, security and crisis management, stress detection, measuring the population's level of happiness, etc. Emotions of all kinds have a profound influence on our day-to-day interactions, affecting our thoughts, behavior and actions. TEM has been a recent established field in computer science and natural language processing which is concerned with the detection, recognition, classification and analysis of human emotions expressed in the text or evoked from the text. The emotions therein expressed have a direct impact on the users' behavior and actions reflected in the social networks subject to the temporal conditions. So, it has become increasingly important to quantify, assess and understand the impact of these emotions on the online social networks [1].

Mining emotions only do not serve the purpose, investigating proper impact analysis would provide even beneficial to latch on the right emotional buttons of users in order to trigger desired emotions deep down into their psyche. This paper also talks about finding impacts of these emotions on various content and user dimensions and thereby focusing on correlational studies. Not only this social emotions expressed in social media networks like Twitter, Facebook etc are also said to have an impact on public discourse and communication in society[2]. Nevertheless, the impact of emotions goes well beyond social networks for example emotional information can be really useful for human-computer interactions and recommender systems, but here impacts of emotions are discussed only in a social media setting.

As TEM is an emerging field so it lacks a view which can put emphasis on the impact analysis studies emerging from this field. The present paper aims to characterize some of the research areas where emotions have proved their influential role so as to provide actionable insights into how to diagnoze and monitor threats and opportunities and track progress against goals. It will also help in developing tailored solutions to instill the right kinds of emotions at the right time. To make the viewpoint of this paper more clear, it will be worth enlisting the various impacts that emotions can produce thereby opening new research avenues. Some of them are:

a) Emotions act as driving force behind the content diffusion in a social media setting. In this way they affect how emotions influence user's information sharing behavior.

b) Emotions developed in a social media setting may affect the emotional states of others, either consciously or unconsciously developing an Emotional Contagion. So, this becomes a hot area of Emotion mining research which studies how emotions impact other users with same or similar emotions which results in shaping public opinions.

c) As emotions are inseparable component of human behavior so their impact on decision making capability of human can never be ignored.

\section{TEXTUAL EMOTION MINING RESEARCH}

Textual Emotion Mining is a very new and promising field of study that attempts to develop automated systems to determine human emotions from text [3]. It is an interdisciplinary field at the cross-roads of information retrieval and computational linguistics. Whenever we talk about any emotion mining system, we observe four core aspects involved in a typical emotion mining application mentioned below (see figure 1).
a. Input
b. Process
c. Output
d. Impact

Being an emerging field, it still lacks a clear view of discoveries that have been made till date and research gaps that 
are yet to be pursued. This manuscript provides an overview of research regarding expressing emotions on social media and their impact on three domains namely, social media content diffusion, emotion contagion and decision making, thereby making recommendations for future research in the area.

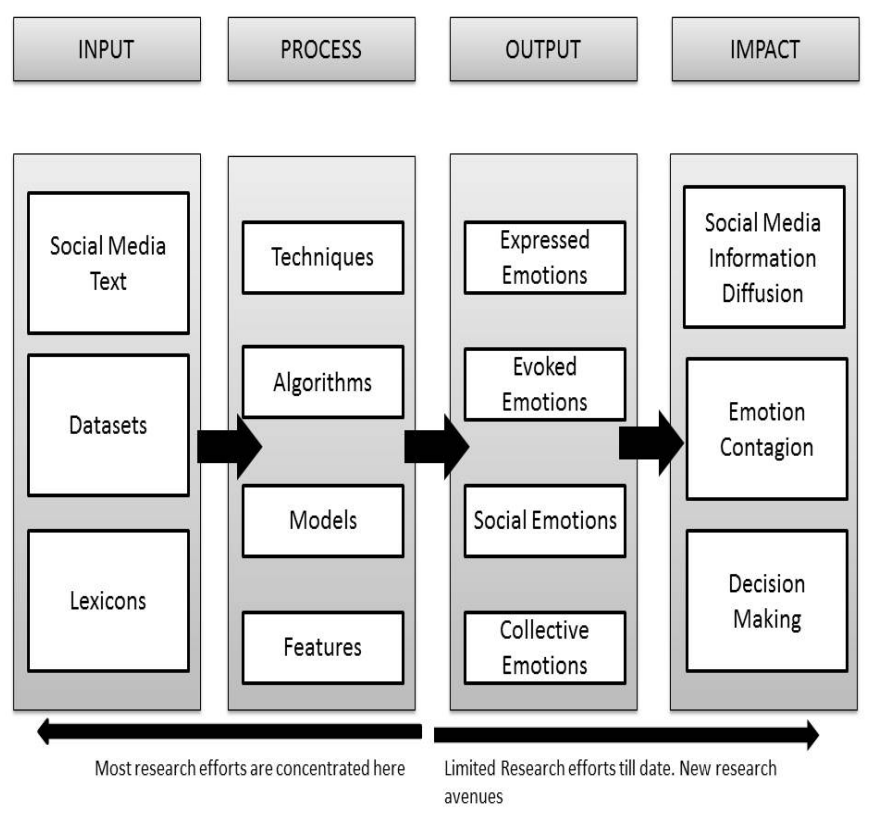

Figure 1: Synthesized view of research on Textual Emotion Mining

This work is an attempt to closely view how to examine and analyze the relationship between output and impact of any emotion mining application. After going through an extensive literature review, it is a generalized observation that most research in this area focuses on developing an effective emotion mining system (i.e., the process aspect) with only a minority progressing towards impact analysis. Categorically, it can be stated that most of the work is being done on the output side to improve it.

There are a few questions dominant regarding the emotional textual interaction between users in online social networks. These are:-

RQ1: What all kinds of emotions are actually involved?

RQ2: How reader's emotions are different from writer's emotions?

RQ3: How exchange of written text creates an emotional influence?

RQ4: What is the true significance of research on Textual Emotion Mining?

RQ5: What are the major areas where emotions create a great impact in a social media setting?

RQ5: How impactful an emotion is over a social group or community, and for how much time?

Through this paper, we would like to seek answers to the above questions in order to understand the true relevance of research in this domain and to shift focus on the areas impacted by emotions like information diffusion, knowledge sharing, influence maximization, the topic of emotions and their role, emotional trust establishment, decision making etc.

\section{PROPOSED MODEL}

The output of any emotion mining system varies in terms of the kinds of emotions being mined. As an answer to the research question 1, four different categories of emotions have been found in the literature, namely expressed emotions, evoked emotions, social emotions and collective emotions. To the best of our knowledge, this manuscript represents the first attempt towards classification of output of TEM. In the present work, we build an emotion-output model to understand the different types of emotions generated during the process of TEM (see figure 2).

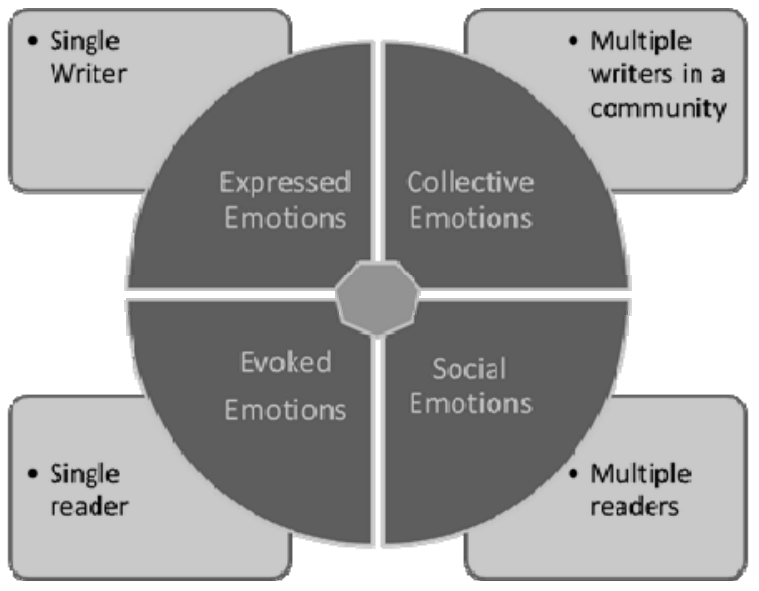

Figure 2: Emotion-Output model

1. Expressed Emotions: The writer/author of the text intentionally or unintentionally embeds his/her emotions into the text while writing a text snippet referred to as expressed Emotions[4]-[8]. The biggest challenge to accurately identify expressed emotions is the use of figurative languages like simile, metaphor, sarcasm, etc . TEM to extract expressed emotions can be treated as a single-label or multi-label classification problem.

2. Evoked Emotions: A reader's mind is triggered with a mix of multiple emotions known as evoked emotions. Emotion mining from the perspective of the reader is generally treated as a multi-label classification problem where the aim is to identify the emotions that is evoked in the minds of a reader.[9]-[15]. Mining readers emotions is a predictive mining task where evoked emotional responses are foreseen and analyzed.

3. Social Emotions: The aggregated form of emotional response given by multiple readers is termed as social emotions. Today, a number of websites have come up with a facility where a number of users can express their evoked emotions through multiple emotion labels after browsing a text segment [16]-[22]. Social emotion detection is naturally a multi-label classification problem where a text is assigned multiple emotional labels by multiple readers. 
4. Collective Emotions: When studied in a community environment, emotions show a collective behavior usually in a conversation i.e., visible correlations can be seen between emotions in consecutive posts of a conversation. Emotions evoked as a result of intra-group interactions over an OSN, affect other members of the ecommunity. Collective emotions portray the categories of emotions which are shared by a large number of individuals at the same time. Chmeil et al [23] studied the collective behavior of on large scale e-communities. Analysis of online data gives us an opportunity to understand the emergence of collective emotions resulting from the interaction of a large number of users with similar emotional interests. Garcia et al [24] proposed an agent-based modeling framework for collective emotions. Tadi et al [25] compiled and analysed a massive dataset containing the dialogs between the users in the MySpace for studying collective behavior of emotions. Abhisheva et al. [26] evaluated the role played by collective emotion dynamics in achieving social polarization in YouTube environment.

\section{IMPACTS}

Forgas once stated that "emotions appear to influence what we notice, what we learn, what we remember, and ultimately the kind of judgements and decisions we make". The differentiated categories of emotions are also found to capable of triggering a short but intense effect on users[27].

\section{A. Information Diffusion}

Users' emotional experiences are closely associated with their information sharing behavior on social media platforms. So, there is a need to understand how emotions impact the dynamics of Information Diffusion. In this regard, we need to answer question like:

- Whether positive emotions spread faster or the negative ones?

- What kind of emotions makes a conversation viral?

- Finding the relation between different categories of emotions and their diffusion speed and quantiy.

Stefan Steiglitz and Xuan[2] examined the effect of emotions on the user's information sharing behavior on social media keeping in track both parameters of diffusion viz, speed and quantity. Ferrara and Yang[28] also assessed the impact of emotions on content diffusion of short text to identify which categories of emotions are more popular in social media communications. A similar piece of research has been conducted in [29] where the authors studied the role of emotions in the context of word-of-mouth marketing, using a dataset of Google+ posts. Guerini et al.[30] presented a thorough analysis of the interplay between evoked emotions and viral facets. Although researchers have focused in this area, but still the understanding of the relationship between emotions and content diffusion in still in its nascent stage and the results can be very promising for gaining business knowledge.

\section{B. Emotional Contagion and Public Opinion Shaping}

Emotions expressed in social media communications are highly contagious in nature and are found to impact the emotional states of other users, resulting to a social network phenomenon known as emotional contagion. Ferrara and Yang[31] demonstrated the dynamics of Emotional contagion on Twitter platform and identified two categories of users based on their susceptibility to emotional contagion. Both the classes are equally likely to adopt positive emotions, however the class of highly susceptible users generally show less inclination towards negative emotions. Kramer et al.[32] proved the evidence of massive-scale emotional contagion over textual communications where he demonstated how emotional states propagate from one user to another over Facebook. This piece of research include experiments on large-scale Facebook user-data to indicate that emotional posts are more engaging. Espinosa and Bernales[33] also focused in the same direction by analyzing the rate of emotion diffusion in Facebook. Xiong et al.[34] proposed an emotional independent cascade model to demonstrate the real-world condition of emotional propagation over heterogeneous social media. Fan et al.[35] aimed their study on prediction of emotional contagion in terms of computer simulation and proposed an agent-based emotion contagion model which is a combination of features of emotion influence and tie strengrth preference in the dissemination process. Different emotions might even compete in shaping the public opinion thereby developing emotional communities[36]. Finding such knowledge will be of great importance for gaining various forms of business intelligence.

\section{Decision Making}

Research on the effect of emotions on people's decision making behavior has started captivating the interests of researchers and business analysts. Online customer reviews and electronic word of mouth(eWOM) are rich sources of emotional content on various e-commerce websites[1]. Relling et al. [37] studied the influence of negative and positive word of mouth (nWOM and pWOM) on customers. Lee et al. [38] assessed the influence exerted by negative emotions in online hotel reviews on consumer's helpfulness perceptions. Another facet of research in this direction include the study of how emotionl content embedded in tweets about a specific company leads to an impact on its future stock prices [39][40].

\section{CONCLUSION}

The Emotion Mining research applies and appeals to every walk of human life. In order to make this technology commercially viable, research groups need to identify the true role of emotions and their possible impacts for the field concerned.

In view of the above-discussed research status and the major impact areas, there is enormous scope of emotion-impact analysis. Apart from that, there is a need for an emotionimpact analysis framework to facilitate advanced analytics. 
With specific focus on emotion-impact analytics, existing solutions in the field are not generic. There is a tight coupling between field-specific analytical solutions. In addition to this, diversity among datasets also exists as a fundamental issue for building generic solutions.

\section{REFERENCES}

[1] H. Hyvärinen and R. Beck, "Emotions Trump Facts : The Role of Emotions in on Social Media : A Literature Review," vol. 9, pp. 1797-1806, 2018.

[2] S. Stieglitz and L. Dang-Xuan, "Emotions and Information Diffusion in Social Media-Sentiment of Microblogs and Sharing Behavior," J. Manag. Inf. Syst., vol. 29, no. 4, pp. 217-248, 2013.

[3] A. G. Shahraki, "Emotion Mining from Text," 2015.

[4] X. Sun, C. Sun, C. Quan, F. Ren, F. Tian, and K. Wang, "Fine-grained emotion analysis based on mixed model for product review," Int. J. Networked Distrib. Comput., vol. 5, no. 1, pp. 1-11, 2017.

[5] N. M. Hakak, "Emotion Analysis : A Survey," pp. 397-402, 2017.

[6] J. Suet and Y. Liew, "FINE-GRAINED EMOTION DETECTION IN MICROBLOG TEXT,” no. May, 2016.

[7] K. Roberts, M. Roach, and J. Johnson, "EmpaTweet: Annotating and Detecting Emotions on Twitter.," Lrec, pp. 3806-3813, 2012.

[8] S. M. Mohammad and S. Kiritchenko, "Using Hashtags to Capture Fine Emotion Categories from Tweets," 2013.

[9] G. Gutu et al., "ReaderBench goes Online : A Comprehension-Centered Framework for Educational Purposes To cite this version : ReaderBench goes On 1 ine : A Comprehension - Centered Framework for Educational Purposes," 2016.

[10] S. Li, J. Xu, D. Zhang, and G. Zhou, "Two-View Label Propagation to Semi-supervised Reader Emotion Classification," Proc. COLING 2016, 26th Int. Conf. Comput. Linguist., pp. 2647-2655, 2016.

[11] R. Xu, L. Ye, and J. Xu, "Reader' s Emotion Prediction Based on Weighted Latent Dirichlet Allocation and Multi-

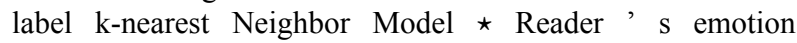
classification and prediction," J. Comput. Inf. Syst., vol. 6, no. 2011, pp. 2209-2216, 2013.

[12] H. Pajupuu, K. Kerge, and R. Altrov, "Lexicon-based detection of emotion in different types of texts: Preliminary remarks," Eesti Raken. Uhin. Aastaraam., no. 8, pp. 171-184, 2012.

[13] S. Bao et al., "Mining Social Emotions from Affective Text," IEEE Trans. Knowl. Data Eng., vol. 24, no. 9, pp. 1658-1670, 2012.

[14] L. Ye, R. F. Xu, and J. Xu, "Emotion prediction of news articles from reader's perspective based on multi-label classification," Proc. - Int. Conf. Mach. Learn. Cybern., vol. 5, pp. 2019-2024, 2012.

[15] Y. Tang and H. Chen, "Mining Sentiment Words from Microblogs for Predicting Writer-Reader Emotion Transition," Proc. Lang. Resour. Eval. Conf., pp. 1226-1229, 2012.

[16] X. Li, Y. Rao, Y. Chen, X. Liu, and H. Huang, "Social Emotion Classification via Reader Perspective Weighted Model.," Aaai, pp. 4230-4231, 2016.

[17] Y. Rao, H. Xie, J. Li, F. Jin, F. L. Wang, and Q. Li, "Social emotion classification of short text via topic-level maximum entropy model," Inf. Manag., vol. 53, no. 8, pp. 978-986, 2016.

[18] F. Krebs, B. Lubascher, T. Moers, P. Schaap, and G. Spanakis, "Social Emotion Mining Techniques for Facebook Posts Reaction Prediction," 2017.

[19] Y. Chang, C. Chu, and C. Chen, "Linguistic Template
Extraction for Recognizing Reader-Emotion," Comput. Linguist. Chinese Lang. Process., vol. 21, no. 1, pp. 29-50, 2016.

[20] J. Lei, Y. Rao, Q. Li, X. Quan, and L. Wenyin,"Towards building a social emotion detection system for online news," Futur. Gener. Comput. Syst., vol. 37, pp. 438-448, 2014.

[21] Y. Rao, Q. Li, X. Mao, and L. Wenyin, "Sentiment topic models for social emotion mining," Inf. Sci. (Ny)., vol. 266, pp. 90-100, 2014.

[22] Y. Rao, Q. Li, L. Wenyin, Q. Wu, and X. Quan, "Affective topic model for social emotion detection," Neural Networks, vol. 58, no. 2012, pp. 29-37, 2014.

[23] A. Chmiel et al., "Collective emotions online and their influence on community life," PLoS One, vol. 6, no. 7, 2011.

[24] D. Garcia, A. Garas, and F. Schweitzer, "Cyberemotions," pp. 187-206, 2017.

[25] B. Tadi, M. Šuvakov, D. Garcia, and F. Schweitzer, “Cyberemotions," pp. 207-229, 2017.

[26] A. Abisheva, D. Garcia, and F. Schweitzer, "When the Filter Bubble Bursts: Collective Evaluation Dynamics in Online Communities," pp. 1-20, 2016.

[27] P. Ekkekakis, The measurement of affect, mood, and emotion: A guide for health-behavioral research. Cambridge University Press, 2013.

[28] E. Ferrara and Z. Yang, "Quantifying the Effect of Sentiment on Information Diffusion in Social Media," pp. 1-15, 2015.

[29] I. Heimbach, B. Schiller, T. Strufe, and O. Hinz, "Content Virality on Online Social Networks," Proc. 26th ACM Conf. Hypertext Soc. Media - HT '15, pp. 39-47, 2015.

[30] M. Guerini and J. Staiano, "Deep Feelings: A Massive CrossLingual Study on the Relation between Emotions and Virality," 2015.

[31] E. Ferrara and Z. Yang, "Measuring emotional contagion in social media," PLoS One, vol. 10, no. 11, pp. 1-14, 2015.

[32] J. E. Guillory et al., "Editorial Expression of Concern: Experimental evidence of massivescale emotional contagion through social networks," Proc. Natl. Acad. Sci., vol. 111, no. 29, pp. 10779-10779, 2014.

[33] K. J. P. Espinosa and A. M. J. Bernales, "Characterizing influence factors affecting emotion diffusion in Facebook," Proc. World Congr. Eng. Comput. Sci., vol. II, pp. 22-24, 2014.

[34] L. Coviello et al., "Detecting emotional contagion in massive social networks," PLoS One, vol. 9, no. 3, pp. 1-6, 2014.

[35] R. Fan, K. Xu, and J. Zhao, "An agent-based model for emotion contagion and competition in online social media," arXiv, pp. 1-22, 2017.

[36] A. Kanavos, I. Perikos, I. Hatzilygeroudis, and A. Tsakalidis, "Emotional community detection in social networks," Comput. Electr. Eng., no. 10, pp. 2515-2525, 2017.

[37] M. Relling, O. Schnittka, H. Sattler, and M. Johnen, "Each can help or hurt: Negative and positive word of mouth in social network brand communities," Int. J. Res. Mark., vol. 33, no. 1, pp. 42-58, 2016.

[38] M. Lee, M. Jeong, and J. Lee, "Roles of negative emotions in customers perceived helpfulness of hotel reviews on a usergenerated review website: a text mining approach," Int. J. Contemp. Hosp. Manag., vol. 29, no. 2, pp. 762-783, 2017.

[39] Hong Keel Sul, A. R. Dennis, and L. I. Yuan, "Trading on Twitter: The Financial Information Content of Emotion in Social Media," 2014 47th Hawaii Int. Conf. Syst. Sci., pp. 806-815, 2014.

[40] H. Kim, I. Son, and D. Lee, "The Viral Effect of Online Social Network on New Products Promotion : Investigating Information Diffusion on Twitter," 지능정보연구, vol. 18, no. 2, pp. 107-130, 2012. 